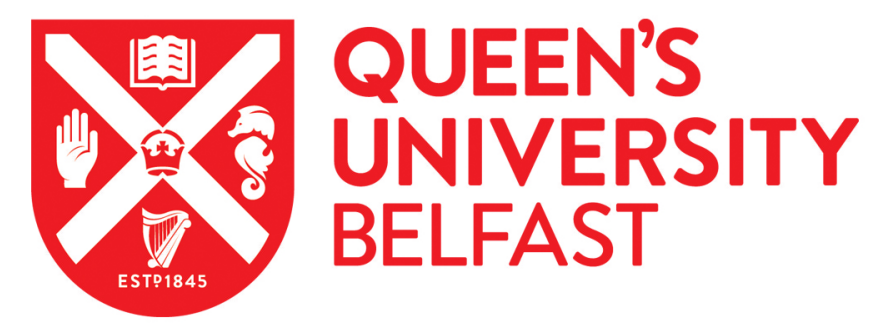

\title{
Evidence of Short-Range Screening in Shock-Compressed Aluminum Plasma
}

Garcia Saiz, M., Gregori, G., Khattak, F., Kohanoff, J., Sahoo, S., Naz, G. S., Bandyopadhyay, S., Notley, M., Weber, R. L., \& Riley, D. (2008). Evidence of Short-Range Screening in Shock-Compressed Aluminum Plasma. Physical Review Letters, 101(7), 075003-1-075003-4. [075003].

https://doi.org/10.1103/PhysRevLett.101.075003

Published in:

Physical Review Letters

Queen's University Belfast - Research Portal:

Link to publication record in Queen's University Belfast Research Portal

\section{General rights}

Copyright for the publications made accessible via the Queen's University Belfast Research Portal is retained by the author(s) and / or other copyright owners and it is a condition of accessing these publications that users recognise and abide by the legal requirements associated with these rights.

Take down policy

The Research Portal is Queen's institutional repository that provides access to Queen's research output. Every effort has been made to ensure that content in the Research Portal does not infringe any person's rights, or applicable UK laws. If you discover content in the Research Portal that you believe breaches copyright or violates any law, please contact openaccess@qub.ac.uk. 


\title{
Evidence of Short-Range Screening in Shock-Compressed Aluminum Plasma
}

\author{
E. García Saiz, ${ }^{1}$ G. Gregori, ${ }^{2,3}$ F. Y. Khattak,,${ }^{1,4}$ J. Kohanoff, ${ }^{1}$ S. Sahoo, ${ }^{1}$ G. Shabbir Naz, ${ }^{1}$ S. Bandyopadhyay, ${ }^{2}$ M. Notley, ${ }^{2}$ \\ R. L. Weber, ${ }^{5}$ and D. Riley ${ }^{1, *}$ \\ ${ }^{1}$ School of Mathematics and Physics, Queen's University of Belfast, Belfast BT7 INN, United Kingdom \\ ${ }^{2}$ Central Laser Facility, Rutherford-Appleton Laboratory, Didcot OX11 OQX, United Kingdom \\ ${ }^{3}$ Clarendon Laboratory, University of Oxford, South Parks Road OX1 3PU, United Kingdom \\ ${ }^{4}$ Department of Physics, Kohat University of Science and Technology, Highway Junction, Kohat, NWFP, Pakistan \\ ${ }^{5}$ Department of Physics, The Ohio State University, Columbus, Ohio 43210, USA
}

(Received 27 February 2008; published 13 August 2008)

\begin{abstract}
We have investigated the angular variation in elastic x-ray scattering from a dense, laser-shockcompressed aluminum foil. A comparison of the experiment with simulations using an embedded atom potential in a molecular dynamics simulation shows a significantly better agreement than simulations based on an unscreened one-component plasma model. These data illustrate, experimentally, the importance of screening for the dense plasma static structure factor.
\end{abstract}

The study of warm dense matter (WDM) is of wide interest with applications to astrophysics, planetary sciences, and fusion science [1,2]. It is of intrinsic scientific interest as the parameter regime it covers (typically densities of $10^{-2}-10^{1} \mathrm{~g} \mathrm{~cm}^{-3}$ and temperatures of $1-100 \mathrm{eV}$ ) is not well understood. The combination of a strong Coulomb interaction between charged particles and partial degeneracy of the electrons means that classical plasma models cannot be applied, while the high temperature precludes the usual solid state physics treatments. Thus, the experimental and theoretical study of WDM presents a significant and worthwhile challenge.

Over the past decade, both angularly and spectrally resolved x-ray scattering experiments [3-6] have been carried out on dense plasma and WDM samples with the aim of accessing information on the static and dynamic structure factors, which themselves are related to fundamental plasma properties [7]. Good experimental data may help to test existing understanding. The theoretical description of x-ray scattering from a dense plasma bears similarities to scattering from a liquid metal and has been discussed in several papers [8-12]. For scattering photons of wavelength $\lambda_{0}$ at an angle $\theta$ the scatter wave vector is given in the nonrelativistic limit by

$$
k=|\mathbf{k}|=\frac{4 \pi}{\lambda_{0}} \sin (\theta / 2),
$$

and the spectrally integrated scatter intensity $I(k)$ for a given scatter wave vector is given as

$$
I(k)=I_{T}(k)\left[\left|f_{I}(k)+\rho(k)\right|^{2} S_{i i}(k)+Z_{f} S_{e e}(k)+Z_{b} S^{\text {inc }}(k)\right],
$$

where $Z_{f}$ and $Z_{b}$ are the number of free and bound electrons, respectively. $I_{T}(k)$ is the classical Thomson cross section. The first term in the square brackets in Eq. (2) describes the elastic scattering and consists of the ionic form factor $f_{I}(k)$, which represents the bound electrons, the screening cloud of free electrons $\rho(k)$ and the ion-ion structure factor $S_{i i}(k)$. The second term, $S_{e e}(k)$, accounts for the spectrally integrated contribution from the free electrons [7]. Inelastic (incoherent) scattering by bound electrons is described by the last term of the equation.

For dense mid- $Z$ elements at modest temperatures, the strong elastic scatter due to bound electrons is easily the dominant term [13], and the angular variation depends on both the atomic (ionic) form factor determined by the electronic charge distribution within the ion and the ionion structure factor which depends on the correlations between the ions. The former is a smoothly varying function decreasing monotonically with angle. The latter can show peaks depending on the degree of coupling between the ions. For a one-component plasma (OCP) model in which ions of charge $Z^{*} e$ are embedded in a rigid uniform sea of negative charge and interact via the bare Coulomb potential [14], the structure factor depends on the strong coupling parameter given in Eq. (3) below:

$$
\Gamma_{i i}=\frac{\left(Z^{*} e\right)^{2}}{R_{i} k_{b} T_{i}}
$$

where $R_{i}$ is the average ion separation, $k_{b}$ is Boltzmann's constant and $T_{i}$ is the ion temperature. The OCP model is expected to apply in the case where electron degeneracy is sufficient to prevent effective screening of the inter-ionic potential. By contrast, we present simulations in this letter, where the inter-ionic potential explicitly includes the effect of a screening cloud of charge around the ions. As we shall see, this significantly alters the predicted scatter structure factor.

Here we present details of an experiment in which a laser-driven shock was used to compress an aluminum $(Z=13)$ foil that was sandwiched between layers of plastic $(\mathrm{CH})$. The plastic layers helped to maintain uniformity of the $\mathrm{Al}$ sample but is not expected to contribute signifi- 
cantly to the elastic scatter due to the low number of bound electrons.

The experiment was carried out using the VULCAN laser at the Rutherford-Appleton Laboratory. Figure 1 illustrates the layout and target. The two shock driving beams were $f / 10$ focus and fitted with phased zone plates (PZP) to create a flat topped focal spot of $1.9 \mathrm{~mm}$ diameter. A further six beams were used as a backlighter. These were focused in an $f / 10$ cluster onto a Ti foil $3 \mu \mathrm{m}$ thick to create an intense source of He-like Ti $1 s^{2}-1 s 2 p^{1} P$ radiation and associated satellites (the $\mathrm{He}-\alpha$ transition) lasting $\sim 1 \mathrm{~ns}$ as measured with an $\mathrm{x}$-ray streak camera. The geometry of the pinhole meant that a narrow $\sim 9^{\circ}$ cone of $x$ rays illuminated the central $1 \mathrm{~mm}$ of the shocked plasma.

All beams were frequency doubled to $532 \mathrm{~nm}$ and were timed to within $\sim 30$ ps of each by use of an optical streak and since they originated from the same oscillator the timing was stable.The beam shape was measured with an optical streak camera and rose in $\sim 130$ ps to a flat top for $1.1 \mathrm{~ns}$ and then fell over $\sim 250 \mathrm{ps}$. The shock drive intensity was $\sim 7 \times 10^{12} \mathrm{~W} \mathrm{~cm}^{-2}$. The coupling of the shock to the $\mathrm{Al}$ foil is potentially affected by the presence of the $\mathrm{CH}$ layer which has a significantly lower density. In order to check this, we performed preliminary experiments in which we only fired the shock drive beams and imaged the rear of the foil out to an optical streak camera.

Using a timing fiducial created by leakage of one of the beams through a mirror we were able to determine the time for shock propagation through the target. This was simu-

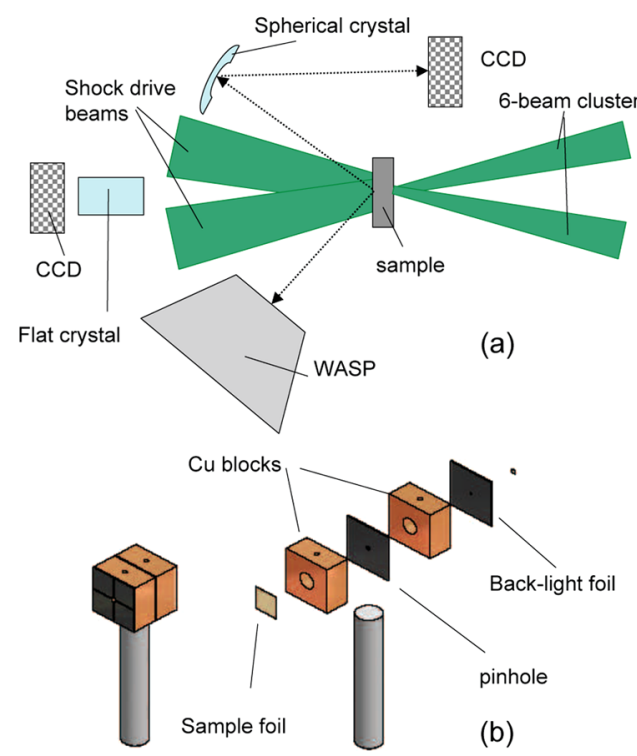

FIG. 1 (color online). (a) Layout of beams and diagnostics for the scatter experiment. Not shown is the optical streak that recorded breakout emission from the rear of a target and the cone used as a a stray light shield for the back-lighter beams (b) construction of a target. The sample foil is $5 \mu \mathrm{m} / 8 \mu \mathrm{m} / 5 \mu \mathrm{m} \mathrm{CH} / \mathrm{Al} / \mathrm{CH}$. The $\mathrm{Cu}$ blocks were $3 \mathrm{~mm}$ thick with $1 \mathrm{~mm}$ holes drilled through and the pinhole was $500 \mu \mathrm{m}$ diameter. lated using the HYADES radiation hydrodynamics code [15], with SESAME equation of state tables [16], and multigroup radiation transport. The time for breakout matched simulation to better than $3 \%$. According to the hydrodynamic simulations this means that the effective irradiance was within $10 \%$ of our expected value and since the equation of state of $\mathrm{Al}$ has been well studied this consistency gives confidence in the simulated plasma conditions. Furthermore, simulations indicated that variation in scatter profiles does not alter our conclusions even for much larger (30\%) changes in irradiance. In our analysis, we use the density and temperatures derived from similar simulations, which were in good agreement with conditions predicted using the QEOS equation of state [17], as an alternative.

The x-ray scatter from the sample was observed with two instruments. One of these was a wide angle spectrometer (WASP) that has been describe elsewhere [18]. It consists of a large HOPG crystal coupled to an image plate. This instrument achieved a spectral resolution of $\frac{\lambda}{\Delta \lambda} \sim 200$, allowing the strong elastic peak to be separated from the much weaker bound-free inelastic scatter. The angular response of the instrument was determined with calibration shots on $\mathrm{Ti}$ and with an $\mathrm{Fe}^{55}$ radioactive source emitting at $5.9 \mathrm{keV}$. This instrument recorded scatter spectra over an $\sim 30^{\circ}$ range and was placed in different positions to cover from $30^{\circ}-85^{\circ}$ during the experiment. The angular resolution was limited to $\sim 10^{\circ}$ mainly by the back-lighter geometry but with a small contribution due to spread of the HOPG crystal.

A quartz spherical crystal spectrometer $(2 d=3.082 \AA)$ was used to observe scatter at an angle of $62^{\circ}$. This instrument achieved a resolution $\frac{\lambda}{\Delta \lambda} \sim 800$ and the elastic peaks from the $1 s^{2}-1 s 2 p^{1} P$ (resonance) and $3 P$ (inter-
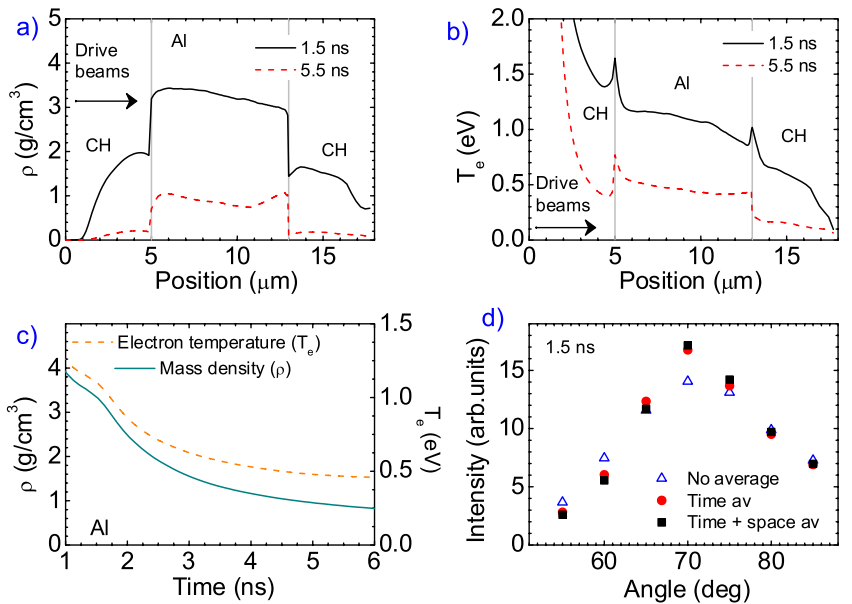

FIG. 2 (color online). (a) Spatial profile of density at 1.5 and $5.5 \mathrm{~ns}$ after the start of the shock driving beams (b) spatial temperature variations at the same times. (c) Spatially averaged density and temperature in the $\mathrm{Al}$ layer as a function of time. The Al layer was divided into 90 cells in the simulations. (d) Comparison of predicted scatter profile for different treatments of averaging over conditions (see text). 
combination) lines were well resolved. A flat crystal spectrometer $[\mathrm{Si}(111)]$ coupled to a $\mathrm{CCD}$ was used to monitor the intensity of the backlighter source through the target. This was used to normalize the scatter signals in the data presented below.

An important factor in the comparison of experiment and theory is the spatial and temporal uniformity of the conditions probed. We can see in Fig. 2 the spatial variation in density and temperature at an early and a late time in the simulation. We can also see a time history of the spatially averaged density and temperature that gives an idea of the role of temporal averaging. In order to check the likely effect of spatial and temporal averaging we have performed simple OCP based calculations in different ways. In Fig. 2(d) the triangles show the result when we run a separate cross-section calculation for each of the $90 \mathrm{Al}$ cells at every $200 \mathrm{ps}$ and average the output cross sections over the foil and $\sim 1$ ns duration of the backlighter. The circles shows the result when we first average the plasma conditions in each cell over $1 \mathrm{~ns}$ and run a cross-section calculation for each cell. Finally, the squares show what happens when we first average the plasma conditions over the whole $\mathrm{Al}$ foil and duration of the backlighter and run a single cross-section calculation. There is clearly a small effect due to averaging, but as we shall see below, it is not great enough to affect our conclusions.

In Fig. 3 we can see how the strength of the elastic scatter peak from the spherical crystal varied with time. The two theoretical curves are calculated using the densities and temperatures from the hydrodynamic simulations. In the OCP calculation, we have taken $Z^{*}=3$, based on the model of Chiu and $\mathrm{Ng}$ [19] to determine $\Gamma_{i i}$ and the ionic form factor. This is then used in the OCP model to determine the ion-ion structure factor, $S_{i i}(k)$.

In the second approach an embedded atom model (EAM) [20] with potential parameters taken from [21], was used in a molecular dynamics (MD) simulation to determine $S_{i i}(k)$ and folded with the same ionic form factor as in the first model. This model has been tested against an ab initio molecular dynamics simulation using a simulation cell containing $32 \mathrm{Al}$ atoms, at a density of $3.7 \mathrm{~g} \mathrm{~cm}^{-3}$ and $T=5050 \mathrm{~K}$, using the CPMD code [22]. The resulting ionion correlation function $g(r)$ and consequently the structure factor, was reproduced very satisfactorily by the EAM. Then, molecular dynamics simulations with the EAM were carried out for a cell containing 864 atoms, at various densities and temperatures. The OCP model indicates a more pronounced peak with time delay than the experimental data: the MD simulation has a less pronounced peak than the data at early time- - but fits better for late time and is at least consistent at all times.

In Fig. 4 we present results at 3 times for the WASP spectrometer. The ordinate is in terms of the reduced wave vector $q=a k$, where $a$ is the average inter-ion distance and $k$ is the scatter wave vector. In these cases, the data are a composite of the data from 2 overlapping angular positions. We assumed that the scaling factor between experi-
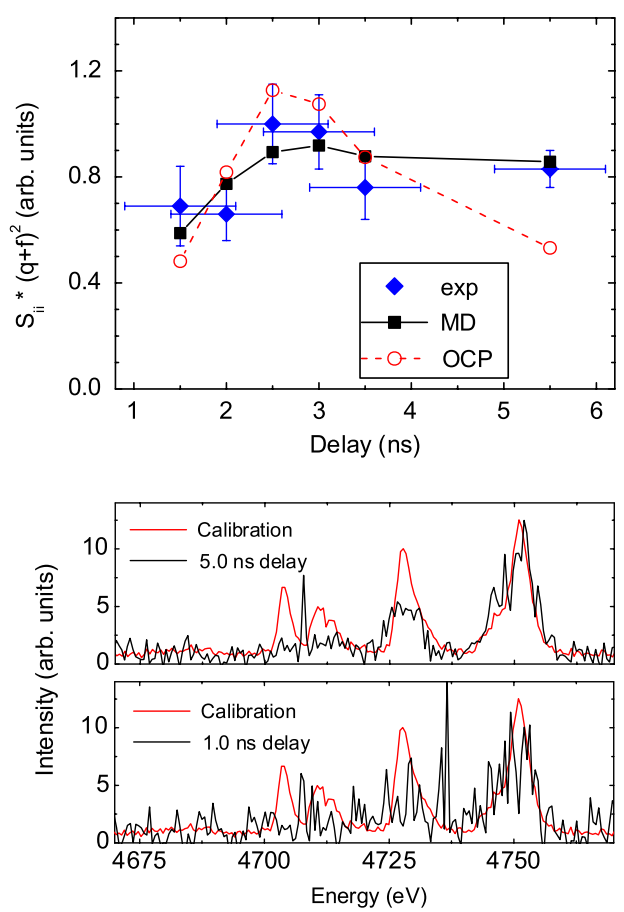

FIG. 3 (color online). Top panel: Relative strength of the elastic X-ray scatter measured by the spherical crystal spectrometer, normalized to back-lighter intensity. The time error bars represent the duration of the backlighter. The theoretical curves are scaled to give the same total signal as the normalized experimental data. Lower panels: examples of the scatter spectra at different times, compared to calibration spectrum.

ment and simulation should be the same for each case and adjusted it to get the best overall fit in each of the two simulation models. We can see that in all cases the EAM based MD simulation is more in accord with experiment than the use of an unscreened OCP model. The rise in signal towards smaller $q$ values was also seen in cold shots (where there was no scatter at $q$ above $\sim 3.5$ ). The rise at low $q$ may be due to the $\mathrm{CH}$ layer, which is not included in the MD based simulations: although calculations using the OCP model, indicated that the $\mathrm{CH}$ layer should contribute very weakly to the signal, as expected from the low number of bound electrons. Looking at the OCP results in Fig. 4 we can understand the peak with time in Fig. 3. At early time, the density is high (small a) and so the reduced wave vector, $q$ is higher than the peak; at late time the reverse is true and the $q$ value seen by the spectrometer sweeps though the peak for a high $\Gamma_{i i}$ plasma as the density falls with time. Screening makes this effect less pronounced in the data and MD simulations.

We expect that screening will not be important when the electrons are very degenerate [14,23]. For the simulated plasma conditions in the $\mathrm{Al}$, we predict $E_{F} / k T \sim 10$ for the data presented. However, we need to look at the Thomas-Fermi screening length to give us an indication of the importance of screening; this is given by [14] 


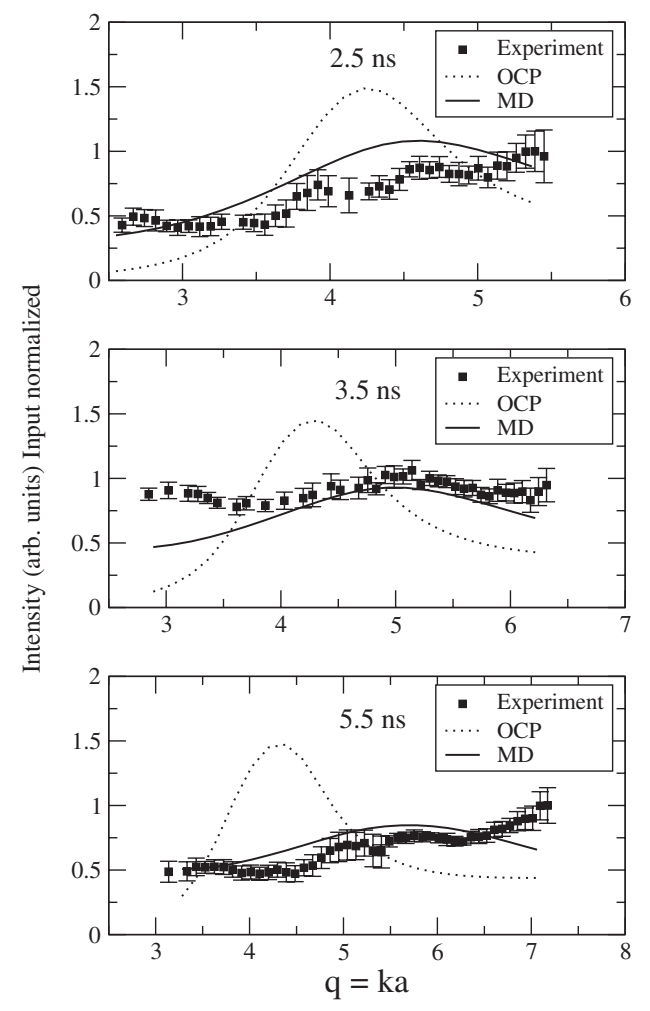

FIG. 4. Comparison of angular scatter data from the WASP spectrometer with MD and OCP simulations of the ion-ion structure factor. Simulations were scaled to data using the same scale factor for all 3 times (see text). Experimental broadening of $10^{\circ}$ was applied to the simulations. The average interion separations are $1.77,2.0$, and $2.28 \AA$ for $2.5,3.5$, and $5.5 \mathrm{~ns}$, respectively.

$$
\frac{\lambda_{\mathrm{TF}}}{a}=\left(\frac{\pi}{12 Z^{*}}\right)^{1 / 3} r_{s}^{-1 / 2}
$$

where $r_{s}=r / a_{0}, r$ is the average electron separation and $a_{0}$ is the Bohr radius. For the average density and temperature trajectories in Fig. 2(c) we expect $\frac{\lambda_{\mathrm{TF}}}{a} \sim 0.25-0.5$. This means that the screening length is somewhat less than the inter-ion separation and we indeed expect that screening effects need to be accounted for in comparison of experiment and simulation. Using $Z^{*}=3$, the data of Fig. 2(c) predicts $\Gamma \sim 80-120$. However, since the potential is modified by a factor, $e^{-r / \lambda_{\mathrm{TF}}}$ the effective strong coupling parameter, $\Gamma^{\prime} \sim 2-10$. We should note here that in previous, double sided compression, experiments [6], we saw a peak narrower than expected in scattering from $\mathrm{Al}$, in contrast to the broad flat peak seen here. However, it is worth noting that in those previous experiments, the thinner targets used meant that the heating was higher and in fact dominated by radiative heating and the narrow peak was seen at late time in the decompressed state. Simulation of such data with the EAM would require more extensive evaluation of the validity of the potential parameters used for higher temperature cases.

In summary, we have used an x-ray scattering experiment to show that for a sample of warm dense matter the inter-ionic screening plays an important role in determining the static structure factor. Although this is expected from the conditions, the fact that we have been able to show this experimentally is a clear advance and demonstrates the power of the X-ray scatter technique.

We would like to acknowledge the support of EPSRC Grant No. EP/C001869/1. G.S.N. is supported by the Higher Education Commission of Pakistan Grant No. 81/S-PAEC/HEC/2004/25.

*Corresponding author. d.riley@qub.ac.uk

[1] Frontiers in High Energy Density Physics-The X-Games of Contemporary Science (National Academies Press, Washington, DC, 2003).

[2] Connecting Quarks With the Cosmos: Eleven Science Questions for the New Century, (National Academies Press, Washington, DC, 2003).

[3] S. H. Glenzer et al., Phys. Rev. Lett. 90, 175002 (2003).

[4] S. H. Glenzer et al., Phys. Rev. Lett. 98, 065002 (2007).

[5] N. C. Woolsey, D. Riley, and E. Nardi, Rev. Sci. Instrum. 69, 418 (1998).

[6] D. Riley et al., Phys. Rev. Lett. 84, 1704 (2000).

[7] S. Ichimaru, Basic Principles of Plasma Physics (Addison, Reading, MA, 1973).

[8] J. Chihara, J. Phys. Condens. Matter 12, 231 (2000).

[9] E. Nardi, Z. Zinamon, D. Riley, and N. C. Woolsey, Phys. Rev. E 57, 4693 (1998).

[10] O. L. Landen et al., J. Quant. Spectrosc. Radiat. Transfer 71, 465 (2001).

[11] G. Gregori, S. H. Glenzer, and O. L. Landen, J. Phys. A 36, 5971 (2003).

[12] A. Höll, R. Redmer, G. Röpke, and H. Reinholz, Eur. Phys. J. D 29, 159 (2004).

[13] E. Nardi, Y. Rosenfeld, and D. Ofer, J. Phys. (Paris), Colloq. 49, C7-267 (1988).

[14] S. Galam and J-P. Hansen, Phys. Rev. A 14, 816 (1976).

[15] J. T. Larsen and S. M. Lane, J. Quant. Spectrosc. Radiat. Transfer 51, 179 (1994)

[16] S.P. Lyon and J.D. JohnsonGroup T-1, Los Alamos National Laboratory Technical Report LA-UR-92e3407 on the internet at http://t1web.lanl.gov/

[17] R. M. More, K.H. Warren, D. A. Young, and G. B. Zimmerman, Phys. Fluids 31, 3059 (1988).

[18] E. Garcia Saiz et al., Rev. Sci. Instrum. 78, 095101 (2007).

[19] G. Chiu and A. Ng, Phys. Rev. E 59, 1024 (1999).

[20] M. S. Daw and M. I. Baskes, Phys. Rev. B 29, 6443 (1984).

[21] J. Mei and J. W. Davenport, Phys. Rev. B 46, 21 (1992).

[22] CPMD V3.11 Copyright IBM Corp 1990-2006, Copyright MPI fuer Festkoerperforschung Stuggart 1997-2001.

[23] F. J. Rogers, Phys. Plasmas 7, 51 (2000). 